Draft version March 4, 2022

Typeset using $\mathrm{LAT}_{\mathrm{E}} \mathrm{X}$ twocolumn style in AASTeX61

\title{
NEUTRAL ISM, LYMAN-ALPHA AND LYMAN-CONTINUUM IN THE NEARBY STARBURST HARO 11 *
}

\author{
T. Emil Rivera-Thorsen, ${ }^{1,2}$ Göran Östlin, ${ }^{1,2}$ Matthew Hayes, ${ }^{1,2}$ and Johannes Puschnig ${ }^{1,2}$
}

${ }^{1}$ Department of Astronomy, Stockholm University, AlbaNova University Centre, SE-106 91 Stockholm, Sweden

${ }^{2}$ Oscar Klein Centre for Cosmoparticle Physics, Stockholm, Sweden

(Received November 24, 2016; Revised January 25, 2017; Accepted January 26, 2017)

Submitted to ApJ

\begin{abstract}
Star forming galaxies are believed to be a major source of Lyman Continuum (LyC) radiation responsible for reionizing the early Universe. Direct observations of escaping ionizing radiation have however been few and with low escape fractions. In the local Universe, only 10 emitters have been observed, with typical escape fractions of a few percent. The mechanisms regulating this escape need to be strongly evolving with redshift in order to account for the Epoch of Reionization. Gas content and star formation feedback are among the main suspects, known to both regulate neutral gas coverage and evolve with cosmic time. In this paper, we reanalyze HST-COS spectrocopy of the first detected local LyC leaker, Haro 11. We examine the connection between LyC leakage and Lyman- $\alpha$ line shape, and feedback-influenced neutral ISM properties like kinematics and gas distribution. We discuss the two extremes of an optically thin, density bounded ISM and a riddled, optically thick, ionization bounded ISM, and how Haro 11 fits into their theoretical predictions. We find that the most likely ISM model is a clumpy neutral medium embedded in a highly ionized medium with a combined covering fraction of unity and a residual neutral gas column density in the ionized medium high enough to be optically thick to Lyman- $\alpha$, but low enough to be at least partly transparent to Lyman continuum and undetected in Si II. This suggests that SF feedback and galaxy-scale interaction events play a major role in opening passageways for ionizing radiation through the neutral medium.
\end{abstract}

* Based on observations with HST-COS, program GO 13017, obtained from the Mikulski Archive for Space Telescopes (MAST). STScI is operated by the Association of Universities for Research in Astronomy, Inc., under NASA contract NAS5-26555. Support for MAST for non-HST data is provided by the NASA Office of Space Science via grant NNX09AF08G and by other grants and contracts. 


\section{INTRODUCTION AND OBSERVATIONS}

Young, star-forming galaxies are believed to be the source of a major part of the radiation which reionized the early Universe. It is however unclear which physical conditions can facilitate the escape of the necessary amount of radiation, given that these galaxies contain large amounts of neutral gas which is opaque to this ionizing radiation at column densities above $\log N \sim 17.2$ (Verhamme et al. 2015). Searches for leaking galaxies at redshifts $z \gtrsim 1$ have yielded few detections (e.g. Iwata et al. 2009; Vanzella et al. 2010, 2012; Nestor et al. 2013; Cowie et al. 2009; Siana et al. 2010), with escape fractions well below the $\sim 20 \%$ needed to account for cosmic reionization (Bouwens et al. 2011; Robertson et al. 2013). A population of lower mass and lower luminosity, star forming galaxies are expected to contribute to reionization, but high star formation is usually coincident with higher neutral gas (surface) density, which would cause a higher probability of blocking the ionizing photons (e.g. Erb 2016; Robertson et al. 2013). In the local Universe, only 9 leakers have been detected so far (Bergvall et al. 2006; Leitet et al. 2011, 2013; Borthakur et al. 2014; Izotov et al. 2016a,b; Leitherer et al. 2016), with escape fractions ranging typically between 1-8\%, with one as high as $f_{\text {esc }} \approx 13 \%$ (Izotov et al. 2016b).

Models of ISM surrounding a central source and allowing escape of Lyman continuum ( $\mathrm{LyC}$ ), span the range of two extremes: In one regime, the surrounding gas is optically thin, highly ionized and density-bounded (see e.g. Jaskot \& Oey 2013), allowing escape for at least a fraction of ionizing photons. In the other regime, the central source is surrounded by an optically thick, ionization bounded medium with the neutral medium surrounding the central Strömgren sphere not completely covering all lines of sight to the background source in what is called the picket fence model (Conselice et al. 2000; Bergvall et al. 2006; Heckman et al. 2011; Zackrisson et al. 2013) or the riddled ionization bounded medium by Verhamme et al. (2015).

The latter paper presents modeling of the imprints of these two extreme scenarios on the observed spectral signature of Lyman- $\alpha$ emission lines, and suggests how these can help point to candidate LyC leakers. The authors compare their theoretical predictions to sample of Lyman- $\alpha$ profiles, including a section of the spectrum treated in this work.

The galaxy treated in this study is Haro 11, a wellstudied Blue Compact Galaxy at low redshift $(z=$ 0.021). Morphologically, it is dominated by three major star-forming knots, called knot A, B and $\mathrm{C}$ respectively, following the terminology of Vader et al. (1993) (see also Kunth et al. 1998; Hayes et al. 2007, and fig. 1). Knots
$\mathrm{B}$ and $\mathrm{C}$ are both very strong in $\mathrm{H} \alpha$, revealing that they also are producing strong $\operatorname{Ly} \alpha$ But while knot $\mathrm{C}$ is a strong Ly $\alpha$ emitter, knot B is a strong absorber, indicating that the neutral ISM properties along the line of sight to the two knots are quite different (Hayes et al. 2009; Östlin et al. 2009). Its complex kinematics indicates that it is undergoing a merger or major interaction event (Östlin et al. 2001, 2015; James et al. 2014). It is the first galaxy in the local Universe reported to leak LyC (Bergvall et al. 2006). The same dataset has since been re-analyzed by Grimes et al. (2007) who concluded that there was no convincing evidence for LyC escape; and since by Leitet et al. (2011) who report an escape fraction of $3.3 \pm 0.7 \%$ based on a new background modeling. In this work, we assume that some radiation does escape, but it should be kept in mind that this result has been disputed. Since Ly $\alpha$ escape is favored by some of the same conditions which also allow LyC escape (see e.g. Hayes 2015; Dijkstra 2014; Leitet et al. 2011), the leaking photons are assumed to originate from knot C, an assumption adopted in this work.

In Rivera-Thorsen et al., submitted to ApJ, we analyzed optical and NUV nebular emission lines in slit spectra from ESO VLT/X-Shooter (see also Guseva et al. 2012). We find from kinematics modelling that both knot $\mathrm{B}$ and knot $\mathrm{C}$ are associated to a component blueshifted by $\sim 50-100 \mathrm{~km} \mathrm{~s}^{-1}$ relative to the mean nebular velocity. This component extends as far as $\sim 200 \mathrm{pc}$. SE of knot B, and $\sim 1.5 \mathrm{kpc} \mathrm{S}$ of the midpoint between the two knots. Given the dense cloud coverage of knot B and the strong star formation activity in both knots, we conclude that this component is not only approaching but also found in front of the starburst regions and thus along the LOS to knot C.

Recently, the galaxy has been observed in $21 \mathrm{~cm} \mathrm{H} \mathrm{I}$ emission with the $100 \mathrm{~m}$. Robert C. Byrd Green Bank Telescope (Pardy et al. 2016). The authors find that Haro 11 has a low gas mass and a very low neutral gas to stellar mass fraction, and that it contains around twice as much ionized as neutral Hydrogen. Interestingly, the authors also find that the bulk of the neutral gas is redshifted relative to the systemic velocity defined from nebular emission from the H II regions surrounding the main starbursts, signifying that the majority of the neutral gas reserves are detached from the star formation activity, possibly a tidal arm being flung outwards as a part of the ongoing merger event.

In this paper, we re-analyze the HST-COS spectrum of Haro $11 \mathrm{C}$ acquired as part of HST program GO 13017 , PI Timothy Heckman. It was first published in Alexandroff et al. (2015) and Heckman et al. (2015) as part of a sample of 22 Lyman Break Analog (LBA) galax- 


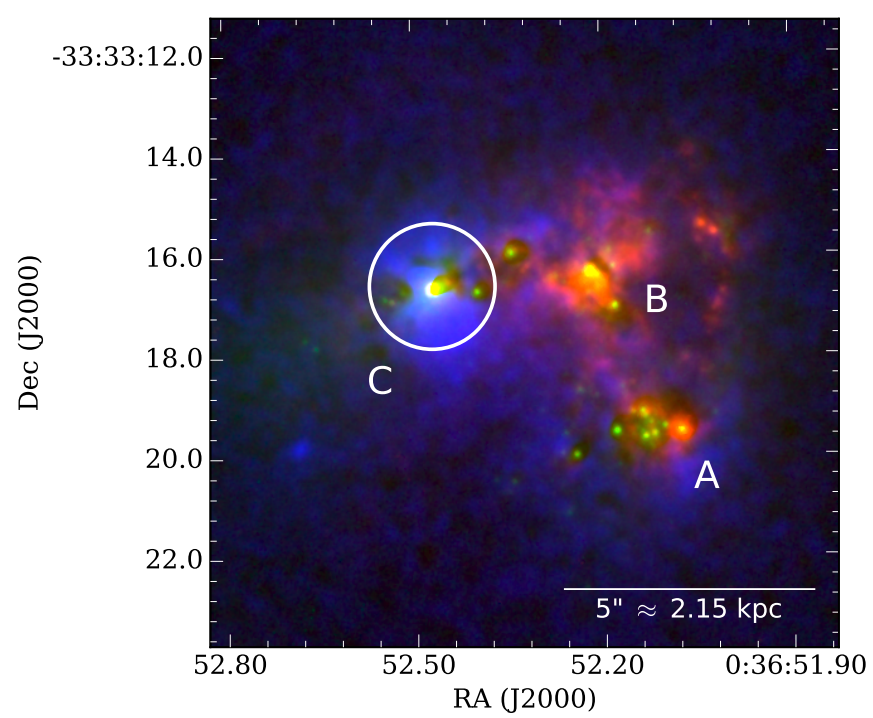

Figure 1. Approximate position of the COS aperture, shown on HST imaging data of Hayes et al. (2009); Östlin et al. (2009), encoding UV continuum in green, $\mathrm{H} \alpha$ in red, and continuum subtracted Lyman $\alpha$ in blue. $\mathrm{N}$ is up, $\mathrm{E}$ is to the left.

ies analyzed individually and as a stack. These authors mainly focus on three indirect indicators of LyC leakage (Overzier et al. 2009; Heckman et al. 2011): Residual flux in the trough of saturated ISM absorption lines, blueshifted emission in Lyman- $\alpha$, and weak optical [S II] emission lines. Details about observation and data reduction are described in depth in Alexandroff et al. (2015); we point the reader there for further information about these. The Ly $\alpha$ profile in this spectrum is, in addition to Heckman et al. (2011), also discussed in Verhamme et al. (2015).

We measure a number of kinematic properties for both the neutral (Low-Ionized State, LIS) and ionized (HighIonized State, HIS) phase. We apply the apparent optical depth method (AOD, Savage \& Sembach 1991; Pettini et al. 2002; Quider et al. 2009; Jones et al. 2013), with the implementation being as described in RiveraThorsen et al. (2015) (RT15) to infer geometric properties of the neutral medium. Under the assumtion that the LyC photons do indeed escape from knot C, we constrain the column density of neutral hydrogen covering the background source.

Fig. 2 gives an overview of the COS spectrum of Haro $11 \mathrm{C}$ treated in this work. Some important features are marked in blue (internal to Haro 11) or orange (Milky Way features).

\subsection{Effective resolution}

For a point source, the resolution of the Cosmic Origins Spectrograph is $R=20,000$, which corresponds to six pixels of the extracted spectrum per resolution element. We have therefore binned the data by a factor of 6 to minimize noise while not losing information. The resolution, however, is generally lower than this for extended sources; for a uniformly filled aperture, it is as low as $R \approx 2,000$. For more morphologically complex sources, the effective resolution depends on the exact shape and angular size of the target.

To estimate the effective resolution for this target, we proceeded as follows. We used imaging data of Haro 11 in the UV-continuum from Östlin et al. (2009); Hayes et al. (2009), since the resolution of absorption lines is determined by the resolution of the background continuum source. We then extracted a circular image at the same position and radius as the COS aperture, which was rotated to match the orientation of the COS as given in the spectrum headers. We modelled vignetting in the COS aperture by multiplying each pixel in the circular UV-continuum image with an interpolation of the values given in the throughput grid in Fig. 7 in Goudfrooij et al. (2010). Finally, the aperture cutouts were collapsed along the cross-dispersion direction, and the FWHM of the flux distribution along the dispersion direction is reported as the effective resolution of this observation. This is converted from arc seconds to COS resolution elements by the factor $0.171 \mathrm{arcsec} / \mathrm{resel}$ reported in the COS instrument handbook (Holland et al. 2014)

The procedure is illustrated in Fig. 3. Here, the upper left panel shows Haro 11 in UV-continuum using HST imaging data from Östlin et al. (2009); Hayes et al. (2009), with the COS aperture coverage from this observation overlaid in blue. The upper right panel shows the galaxy as the COS saw it, with the region covered by the aperture cut out, rotated and vignetting-corrected as described above. Finally, the lower panel shows the flux profile in the aperture, collapsed along the cross dispersion direction. The FWHM of this distribution has been adopted as the effective resolution for this observation. We find $R_{\text {eff }}=0.3^{\prime \prime}=1.75$ spectral resolution elements as defined in the COS instrument handbook Holland et al. (2014), which at the wavelength of observed Ly $\alpha$ corresponds to $31 \mathrm{~km} \mathrm{~s}^{-1}$. This value might however be slightly underestimated, since the flux profile is not well described by a Gaussian profile, but has stronger wings due to the morphology of the target in the aperture, meaning that a larger fraction of the total flux is outside the FWHM.

\section{ANALYSIS}

\subsection{Individual lines}




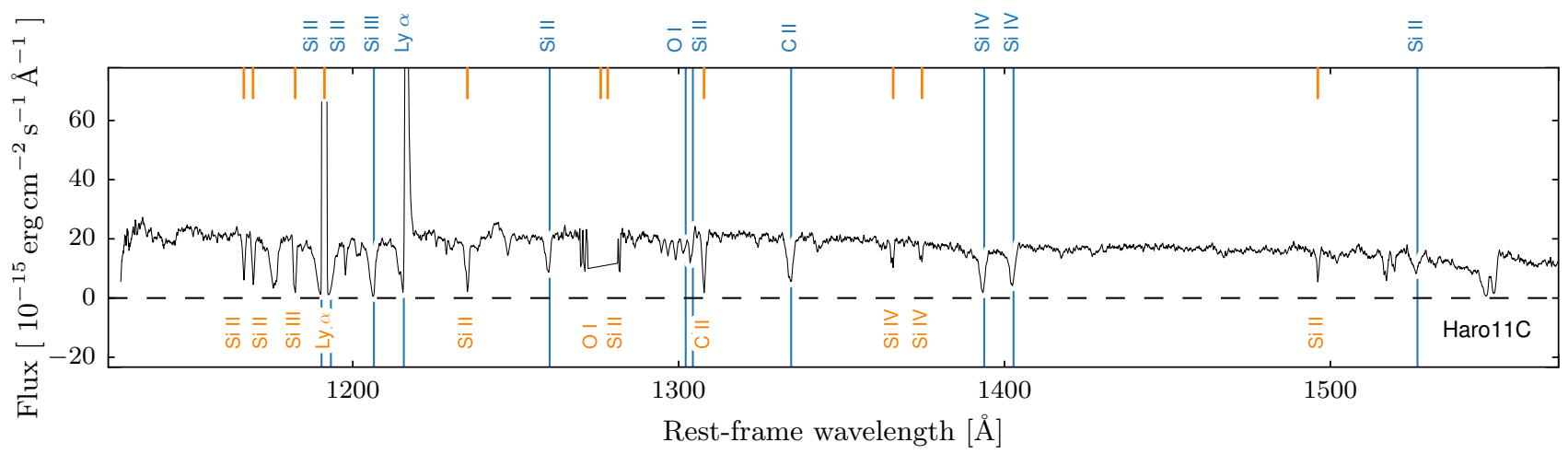

Figure 2. Overview of the relevant regions of the COS spectrum of Haro $11 \mathrm{C}$, shown in rest frame wavelengths of the object. Some key spectral lines are marked; Milky Way features in orange, features of Haro 11 in blue.
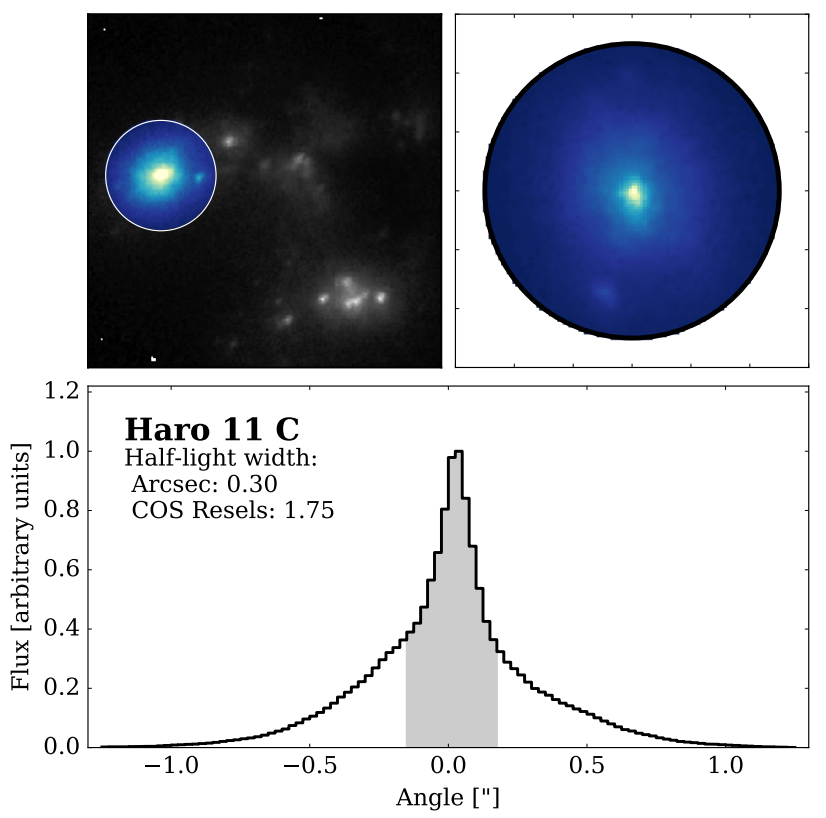

Figure 3. Effective resolution estimate. Upper left: Haro 11 in UV-continuum, data from Östlin et al. (2009); Hayes et al. (2009). Data is shown on a square root scale and cut levels set for best detail. Inset in blue is the COS aperture covering the region surrounding knot $\mathrm{B}$ in the terminology of Vader et al. (1993). $\mathrm{N}$ is up, E is left. Upper right: The extracted, throughput-corrected in-aperture image, rotated so horizontal corresponds to the dispersion direction of the spectrograph. Lower panel: The collapsed flux profile in the aperture, with the shaded region showing the width of the effective resolution element, which is also given in arc seconds and resolution elements/bins.

Figure 4 shows the individual profiles of the transitions included in our analysis; the upper panel shows transitions of Si II, lower panel of Si IV. It is plainly visible that the ionization fraction is high, with the Si IV curves being considerably deeper than the low-ionized lines. Looking at the upper panel, Si II $\lambda \lambda 1304,1526$ are

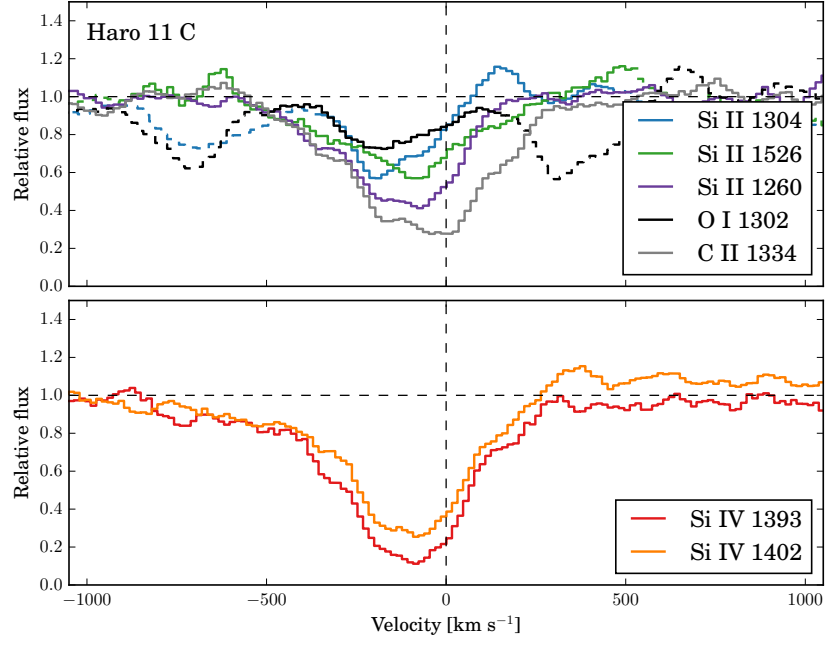

Figure 4. The Si II and other LIS (upper) and Si IV (lower) profiles included in this study. Dashes denote data points masked out due to foreground contamination. Data in this figure has been smoothed to better show the line shapes, but all computations have been carried out on the unsmoothed data. Note the high-velocity component in both Si IV lines, extending out to $\sim 850 \mathrm{~km} \mathrm{~s}^{-1}$.

somewhat shallower than Si II $\lambda 1260$. The former two lines have comparable oscillator strengths, both about a factor of 10 lower than that of $\lambda 1260$. It is thus clear that we do not find ourselves in the optically thin regime, in which the latter line should be correspondingly around 10 times stronger; on the other hand, it is possible that the two weak lines are not completely saturated. In the lower panel, the two Si IV lines have oscillator strengths within a factor of 2 of each other. They are thus at first glance consistent with a medium that is not completely opaque, but not with an optically thin one, and within uncertainties consistent with the optically thick. The stronger absorption in Si IV reveals a high level of ionization of the medium covering the background source. 
Table 1. Measured properties

\begin{tabular}{lc}
\hline \hline \multicolumn{1}{c}{ Quantity } & Haro $11 \mathrm{C}$ \\
\hline$z^{\mathrm{a}}$ & $0.02043 \pm 0.00002$ \\
$z_{v}\left[\mathrm{~km} \mathrm{~s}^{-1}\right]^{\mathrm{a}}$ & $6126 \pm 7$ \\
$\Delta v^{\mathrm{LIS}}$ & $480 \pm 15$ \\
$v_{\text {int }}^{\text {LIS }}$ & $-149 \pm 7$ \\
$v_{95 \%}^{\text {LIS }}$ & $-421 \pm 12$ \\
$v_{\text {peak }}^{\text {Ly }}$ & $158 \pm 0.8$ \\
$\log _{10}\left(N_{\mathrm{SiII}}\right)^{v=0}$ & $12.1 \pm 0.2$ \\
\hline
\end{tabular}

${ }^{\mathrm{a}}$ From Sandberg et al. (2013)

\section{2. $N_{\mathrm{Si}}$ and $f_{C}$}

Following the method described in RT15, we have performed fits for column density and covering factor in each velocity bin, for both the high- and low-ionization state. Here we shall briefly summarize the method, but refer to RT15 and references therein for a detailed explanation.

In any given velocity bin, the residual line intensity in terms of the continuum intensity is given as

$$
\frac{I}{I_{0}}=1-f_{C}\left(1-e^{-\tau}\right),
$$

with the optical depth $\tau$ given as:

$$
\tau=f \lambda \frac{\pi e^{2}}{m_{e} c} N=f \lambda \frac{N}{3.768 \times 10^{14}}
$$

Here, $f$ is the oscillator strength of a given transition, $\lambda$ is its rest frame wavelength in $\AA, N=N(v)$ is the column density of the relevant ion within the given velocity bin, and $f_{C}$ is the covering fraction of neutral gas in same velocity bin. When multiple absorption lines are present which arise from the same ground state; the population of this state is the same for all transitions, and their relative strengths are governed simply by their oscillator strengths, and with two or more such transitions, $f_{C}$ and $N$ can be inferred from knowledge of $f \lambda$ and measured values of $I / I_{0}$.

The method is illustrated in Fig. 5: In the upper panel is shown the line profiles of the three Si II transitions included in the analysis. The red vertical line marks the zero-velocity bin. From this bin, the three relative fluxes are plotted in the lower frame against their wavelength scaled oscillator strengths $f \lambda$ on the $x$ axis. In magenta is shown the best fit of the function $I / I_{0}(f \lambda)$ described above. Also shown are two examples of different parametrizations of this function, to show how the
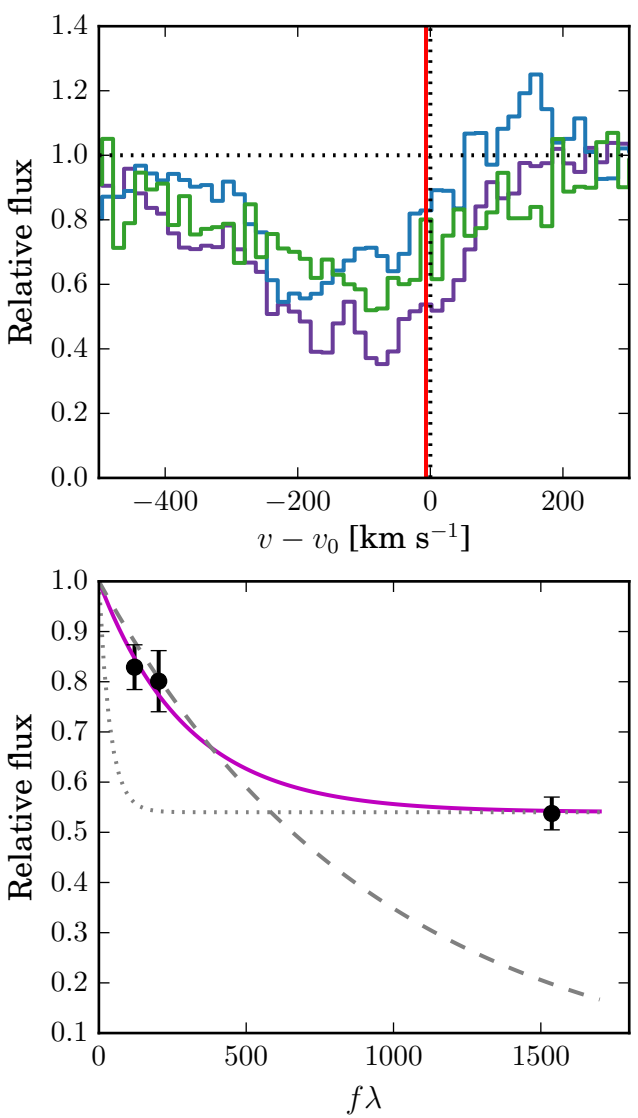

Figure 5. Illustration of the Apparent Optical Depth computations. Upper panel: the three Si II lines in this analysis shown together, with the zero-velocity bin marked with a vertical red line. Lower panel: From this bin, the three measured relative intensities are plotted against $f \lambda$ as black dots, with vertical error bars showing the error spectrum from the COS pipeline. The best-fit function $I / I_{0}(f \lambda)$ as defined above is drawn in magenta. Also shown for illustration are the two extreme examples of a very high column density and unchanged covering fraction, and of a very high covering fraction but significantly lower column density.

two parameters influence its shape. One, in dotted gray, shows what happens to the best-fit function when $N$ is raised strongly, while $f_{C}$ is kept unchanged, while the other in gray dashes shows the curve for a $f_{C} \approx 1$ and $N$ is 0.5 dex below the best-fit value. This is of interest to the discussion of radiative transfer effects below in sect. 3.4.

The resulting values of $N_{\mathrm{SirI}}$ and $f_{C}$ are shown in fig. 6 . The upper panels show the pseudo-reduced $\chi^{2}$ as defined in $\operatorname{RT} 15\left(=\chi^{2} /(\mathrm{DOF}+1)\right)$ for each bin, middle panels show the inferred column density in each bin, with surrounding shaded columns showing the confidence intervals. In the lower panels, the mean LIS line profile is shown in black with gray shaded uncertainty inter- 
vals. On these are overlaid the best-fit values of $f_{C}$ as colored dots, with surrounding shaded bars showing the confidence intervals. We again caution that $f_{C}$ is the covering fraction of neutral gas within the given velocity bin, and hence only provides a lower limit for the total, geometric neutral gas covering fraction, since gas at different velocities generally does not occupy the same projected area.

\section{DISCUSSION AND CONCLUSIONS}

\subsection{Lyman- $\alpha$ and ISM absorption profiles}

In fig. 7 , we show the neutral and ionized absorption profile as in fig. 6 together with the profile of Ly $\alpha$ on a common velocity scale.

The picture is what we would expect from a LyC leaker: The neutral absorption features are weak, and the ionized features are strong, revealing a highly ionized medium in front of the central cluster. This is also in good agreement with the findings of Pardy et al. (2016), who conclude that the galaxy has about twice as much ionized as neutral gas. In addition to this, it is interesting to note the close similarity in shape between the Si II and Si IV line profiles, indicating that they likely represent two different phases in the same higher density regions. These regions will likely be ionized on the side facing the central cluster, being photoionized by its hot, massive stars. This also implies that the nebular emission outflow found in Rivera-Thorsen et al, submitted, at least partially traces the same gas as the Si IV absorption here and, by extension, also the neutral medium. Looking at fig. 4, we see that maximum absorption and thus the largest amount of gas in a single velocity bin for both phases is found at around $v \approx-50 \mathrm{~km} \mathrm{~s}^{-1}$. Interestingly, a component was also found in nebular emission at this velocity in the observations of Rivera-Thorsen et al., submitted. The outflow velocity $v_{\text {int }}$ is at $149 \pm 7 \mathrm{~km} \mathrm{~s}^{-1}$, fully consistent with the findings of Heckman et al. (2015) and Alexandroff et al. (2015). Interestingly, Sandberg et al. (2013) found from the neutral Sodium resonance absorption doublet $\lambda \lambda 5889.95,5895.92(\mathrm{NaD})$ an overall, weak redshift of $v=32 \mathrm{~km} \mathrm{~s}^{-1}$. From e.g. fig. 7, it is evident that while neutral gas is present at these velocities, the velocity is at odds with the integrated velocity of $v=-149$ $\mathrm{km} \mathrm{s}^{-1}$ found in this work. We note that $\mathrm{Na}$ I has a very low ionization potential of only $\sim 5 \mathrm{eV}$, meaning that these atoms may well only be present in the densest and/or dustiest regions, in which Sodium is shielded from ionization. This is in agreement with the finding of Sandberg et al. (2013) that NaD, despite being a strong transition, shows absorption of only $\sim 95 \%$ of continuum level and is mostly found in small, optically thick clouds.
This suggests that the NaD is tracing only the densest and/or dustiest regions which are more slowly accelerated by star formation feedback than the surrounding, more dilute medium. In fig. 1, dusty regions in the aperture are apparent $\mathrm{E}$ and $\mathrm{W}$ of knot C. Seen in the better resolution of fig. 1 in Adamo et al. (2010), these regions seem like they might be connected by a narrow dust lane partially covering the background source. We speculate that the NaD absorption of Sandberg et al. (2013) might be associated with this.

Also the absorption feature in the Lyman $\alpha$ profile in the upper panel seems to morphologically follow the shape of the metal lines, indicating that radiative transfer effects are modest, indicative of a fairly low column density of H I around line center. We find a Lyman $\alpha$ peak velocity of $v_{\text {peak }}^{\text {Ly } \alpha}=158 \pm 1 \mathrm{~km} \mathrm{~s}^{-1}$ relative to the systemic velocity found by Sandberg et al. (2013), derived from nebular emission lines in the region around knot C. This velocity, as it is also discussed in Verhamme et al. (2015), is just consistent with their theoretical predictions for a density-bounded, low-column density system, albeit on the upper limit of their allowed range.

\subsection{Metal and H I column density}

Lyman $\alpha$ escape is mainly governed by gas at or near systemic velocity. In the middle panel of fig. 6 (a) is shown the best-fit column density $N_{\mathrm{SiII}}$ for each velocity bin. The value at systemic velocity is $\log _{10}\left(N_{\mathrm{SiII}}\right)=$ $12.1 \pm 0.2$. We adapt the value for local starbursts of $\log (\mathrm{Si} / \mathrm{O})=-1.59 \pm 0.07$ from Garnett et al. (1995), and use this to estimate the column density of neutral Hydrogen in front of the light source in the same way as in Puschnig et al. (submitted to MNRAS) as follows. Guseva et al. (2012) found a metallicity of Haro $11 \mathrm{C}$ of $12+\log _{10}(\mathrm{O} / \mathrm{H})=8.1$. With $\log _{10} \mathrm{Si} / \mathrm{O}=-1.59 \pm 0.07$, this leads to a $\mathrm{Si} / \mathrm{H}$ ratio in the neutral medium of $\mathrm{Si} / \mathrm{H}$ $=3.24_{-0.48}^{+0.57} 10^{-6}$, leading to a Hydrogen column density of $6.2_{-1.1}^{+0.9} \times 10^{17} \mathrm{~cm}^{-2}$ for just one velocity bin. Since $\mathrm{H}$ I gets opaque to ionizing radiation at $\log N \sim 17.2$ (Verhamme et al. 2015), this range is not consistent with the low- optical depth, density bounded scenario.

Furthermore, while Ly $\alpha$ radiative transfer is dominated by gas of $v \sim v_{0}$, LyC is sensitive to $\mathrm{H} \mathrm{I}$ at all velocities. The total column density of Si II depends on the configuration of the neutral clouds. Assuming that the area covered by gas in each velocity bin is completely randomly located, a lower limit to the total column density is:

$$
N_{\text {SiII }}^{\text {mean }}=\sum_{i} N_{\mathrm{HI}, i} f_{C, i}
$$

Summing this over $-450 \mathrm{~km} \mathrm{~s}^{-1}<v<150 \mathrm{~km} \mathrm{~s}^{-1}$, the velocity range over which the column densities can 


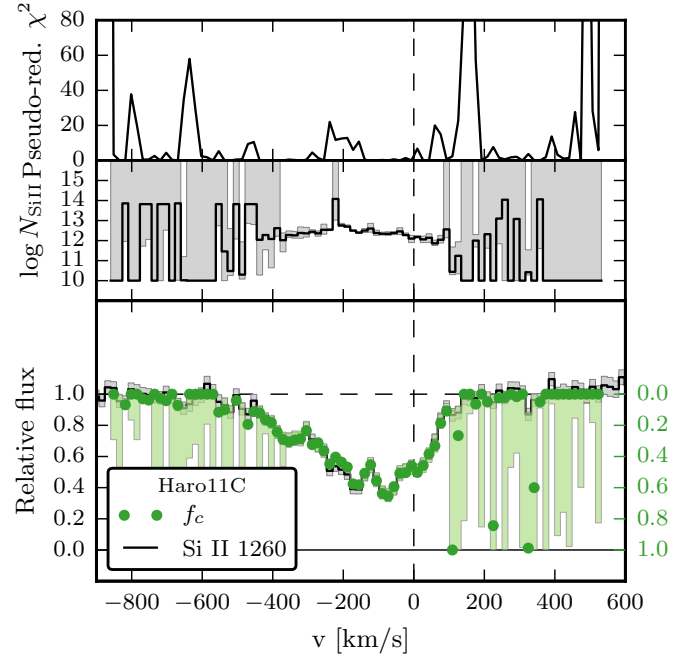

(a) LIS

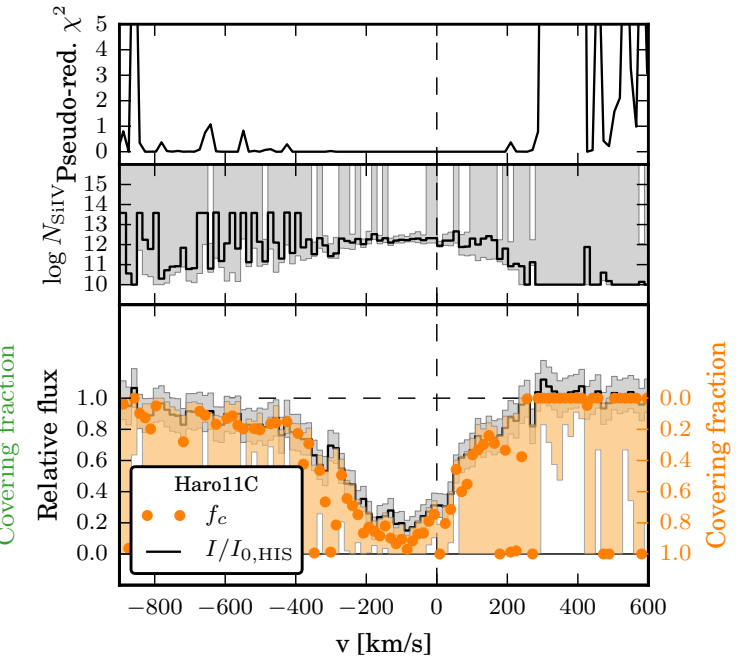

(b) Si IV

Figure 6. Upper panels: Pseudo-reduced $\chi^{2}$ as described in RT15. Middle panels: Best-fit ion column density with confidence intervals in shaded gray. Lower panels: Si II $1260 /$ meanSi IV profile shown as black steps, with inferred $f_{C}$ shown with yellow dots. Lighter shaded columns show confidence intervals for both.

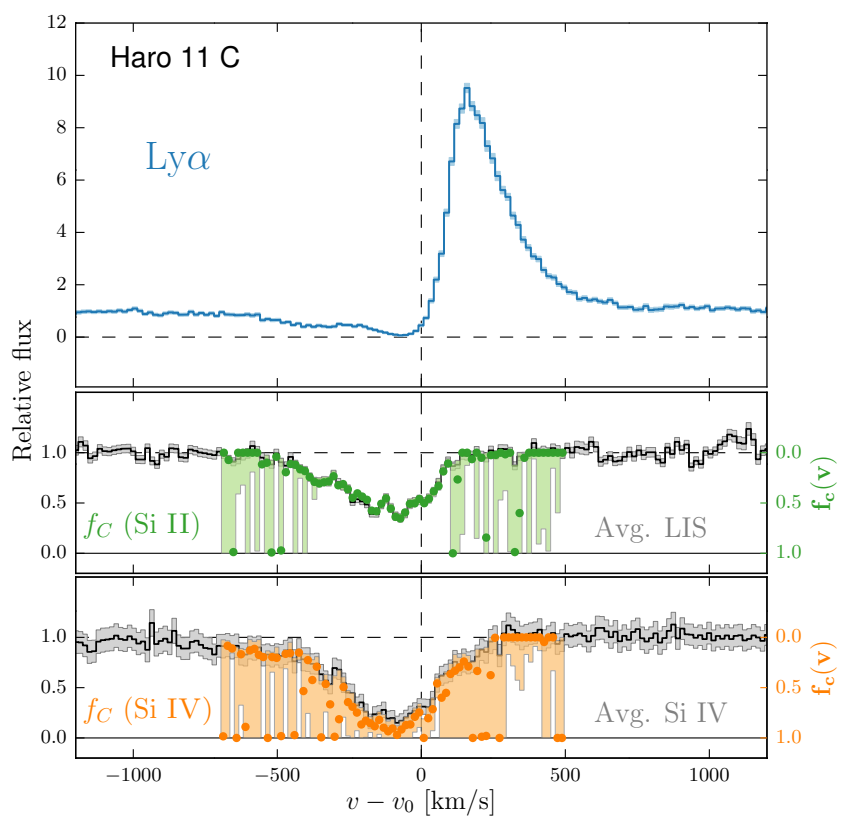

Figure 7. Upper panel: Ly $\alpha$ profile of Haro $11 \mathrm{C}$, in approximate units of the surrounding continuum level. The full line indicates the measured values smoothed by a 5 px. flat kernel; surrounding shading encloses the $\pm 1 \sigma$ error band (mostly comparable in width to the line width). Middle panel: Black steps show the averaged LIS line profile, smoothed by a 5px kernel. Surrounding gray shading denotes the $\pm 1 \sigma$ confidence band. Lower panel: Same as middle panel, but for the Si IV transitions. be reasonably well determined (and removing the unphysically high value in the bin at $v \approx 222 \mathrm{~km} \mathrm{~s}^{-1}$ ), yields a lower limit of $\log N_{\mathrm{SiII}}=13.52 \pm 0.15$ and $\log N_{\mathrm{HI}} 19.01 \pm 0.17$, corresponding to $\approx 64$ optical depths in LyC, strongly incompatible with an optically thin, density-bounded scenario.

However, the strong riddled ionization bounded scenario - i.e. consisting of only a fully neutral and a fully ionized phase - is easily ruled out since the Ly $\alpha$ profile does not have any appreciable emission component at zero velocity. We therefore expect a residual neutral fraction to remain in the ionized phase; a fraction which has a column density high enough to block Ly $\alpha$ efficiently at line center, but low enough to be at least part transparent to LyC radiation and undetected in Si II.

We can estimate the lowest detectable $N_{\text {SiII }}$ by noting that the relative errors for the Si II lines in our spectrum are $\sim 0.05$. Assuming a covering fraction of unity for the dilute neutral component, and adopting the oscillator strength of the strongest of the Si II transitions, $f \lambda_{1260}=1486.8$, we find in the limit that $I / I_{0}=e^{-\tau}=0.95$, and eq. 2 becomes:

$$
N_{\mathrm{SiII}}^{\min }=-\log _{e}(0.95) \frac{3.768 \times 10^{14}}{f \lambda}=10^{10.1} \mathrm{~cm}^{-2},
$$

which with the adopted metallicity for Haro $11 \mathrm{C}$ corresponds to a minimum Hydrogen column density of $N_{\mathrm{HI}}^{\min } \sim 4.0 \times 10^{15} \mathrm{~cm}^{-2}$. This leaves around 2 orders of magnitude in $N_{\mathrm{HI}}$, in which the gas is not detected in Si II and is optically thick to Ly $\alpha$ while translucent to LyC. If we require the gas to be detected in at least 
two of the lines included in this analysis, the limiting hydrogen column density becomes $N_{\mathrm{HI}}^{\min } \sim 4.9 \times 10^{16}$ $\mathrm{cm}^{-2}$, adding another order of magnitude to the allowed range, but we adopt the lower value as a conservative estimate.

The existence of a diffuse neutral component being present in the ionized medium seems consistent with what is found in Lyman Break Analogs and Green Pea galaxies (Heckman et al. 2011; Henry et al. 2015). These galaxies have sometimes very low LIS absorption depths, which is usually indicative of low covering fractions and high porosity of the neutral medium - and yet they find covering fractions of near unity of $\mathrm{H}$ I from Lyman $\beta$ absorption, indicating column densities of $N \gtrsim 10^{16}$ $\mathrm{cm}^{-2}$. Like in the case of Haro 11, this indicates that a non-negligible neutral component must be present in the ionized phase, although it may very well be of such low densities and metallicity that metal absorption from this medium is undetectable.

The column densities we derive for Si II in this work are generally between $12.0 \lesssim \log N \lesssim 12.5$, column densities around which the transitions involved become optically thick: For $\lambda \lambda 1260,1526$ and 1304, $\tau$ becomes 1 at $\log N \sim 11.3,12.2$ and 12.5 , respectively. Off the regions of strongest absorption, and in particular at $v_{0}$, only $\lambda 1260$ seems to be optically thick, and it seems the column densities arrived at can be trusted. But at line center, around $v \sim v_{\text {int }}$, the column densities found are so close to the limit that they are most likely to be interpreted as lower limits. There might also be systematics in the determination of the continuum around the lines which may lead to the lines at $\lambda \lambda 1304,1526$ to be falsely seen as shallower than $\lambda 1260$, meaning that all computed column densities are really lower limits rather than actual values. The confidence intervals in fig. 6 do not reflect this possible saturation, but only the formal errors from the best approximation to the residual intensity, they do not include systematics.

If the computed column densities are in fact lower limits, this means the inferred $\mathrm{H}$ I column densities are also lower limits. This would strengthen the modified riddled, ionization-bounded medium scenario that the ISM on the line of sight to Haro $11 \mathrm{C}$ consists of dense, neutral clumps with an ionized interclump medium containing a dilute neutral component.

\subsection{Neutral gas metallicity}

The exact value of the metallicity is however uncertain. The values found from nebular recombination lines by Guseva et al. (2012) are measured mainly in the central H II regions around the clusters; and differ by 0.2 dex between knot B and C. The neutral, outflowing gas

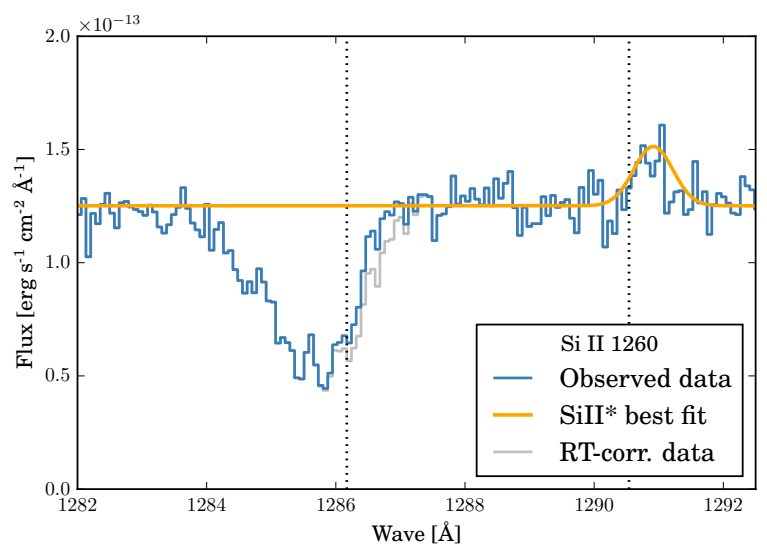

Figure 8. Si II $\lambda 1260$ and its fluorescent line at $\lambda 1265$, with the observed data shown in blue. The orange curve shows a fit to the fluorescent emission, and in gray is shown the worst-case absorption profile corrected for radiative transfer effects re-filling the absorption trough.

could be mixed, or have an unseen LOS distance component larger than the knot separation, drawing into question which is the better value to assume for this gas. We base our conclusions on the value found for knot $\mathrm{C}$, but note that using the metallicity of $12+\log (O / H)=8.3$ found for knot B by Guseva et al. (2012), the inferred H I column densities are $3.9 \pm 0.6 \times 10^{17} \mathrm{~cm}^{-2}$. However, the question of the exact metallicity of the outflowing gas is complicated. Gas closer to the star forming regions is expected to be more strongly enriched than gas further away, which would imply that the $\mathrm{H}$ I column density is larger than inferred from Si II above. To this can be added the further complication stemming from the merger event that the galaxy is currently undergoing, which may have mixed gas of different metal contents. In any case, though, we would expect the regions nearest to the starbursts to generally have higher metallicity than the surrounding cool gas, such that the HI column density inferred above is more likely to be underestimated than overestimated.

\subsection{Radiative transfer effects}

The conclusions drawn by the AOD method rest on the assumption that the observed lines are close to being pure absorption lines, with redistribution of photons due to radiative transfer effects being modest. Modelling work by e.g. Prochaska et al. (2011) and Scarlata \& Panagia (2015) has shown that in certain conditions, radiative transfer can re-fill absorption features in a way that can make an isotropic, optically thin medium mimic the observational fingerprints of a system of optically thick clumps. We therefore need to investigate whether our conclusions could be generated by such effects. 
Because the ground level in Si II is a doublet with a short-lived upper level, each of the Silicon lines included in the analysis has a fluorescent emission companion with practically no absorption component. The fluorescent line can be used to constrain the possible effect of radiative infilling of the absorption trough. If each photon in the fluorescent transition on average has scattered once in the neutral medium, the ratio of emission in this line and re-filling of the resonant absorption line is simply that of their Einstein coefficients $A_{k i}$. For each subsequent scattering, more photons escape directly through the fluorescent channel, and the infilling of the absorption line is further suppressed.

In fig. 8, the observed line profile is shown in blue, along with the best fit to Si II* shown in orange. Since the fluorescent and resonant reemission originate in the same environments, the shape of the resonant reemission profile can be completely determined from the shape and center of the fluorescent line and the assumption of one scattering. It is therefore simple to construct this theoretical reemission line, subtract it from the observed absorption line and find the limiting depth of the pure absorption line without reemission; this modeled line is shown in gray in the figure. It is readily seen that the difference between worst-case intrinsic and observed line is so small that it cannot affect our conclusions about the covering factors and column densities of the neutral gas significantly.

We have not performed similar measurements for the lines at $\lambda \lambda 1304,1526$, but note that if significant refilling is present here, this would mean the column densities had been underestimated in our analysis, which would strengthen the conclusions of a clumpy medium optically thick in Si II 1260, but part transparent in the much weaker other lines.

\subsection{II $\lambda 1334$ absorption}

Looking back at Fig. 4, the profile of C II $\lambda 1334$ is clearly deeper than Si II $\lambda 1260$, which we had otherwise concluded is optically thick and thus provides a limit for the velocity binned covering fractions. We believe the explanation is a contribution from C II $\lambda 1335.7$ blending with $\lambda 1334$. This transition has an oscillator strength of $f_{i k}=0.114$, compared to $f_{i k}=0.127$ for $\lambda 1334$ (there is a third line at $1335 \AA$, but that is an order of magnitude weaker), so given a sufficient population of its ground level, it is strong enough to make a non-negligible contribution to the resulting line profile. One should however bear in mind that these lines arise from different lower fine structure levels; $\lambda 1334$ arises from ${ }^{2} \mathrm{P}_{1 / 2}$, while the other two arise from ${ }^{2} \mathrm{P}_{3 / 2}$. The population of the latter level depends sensitively on physical conditions in its

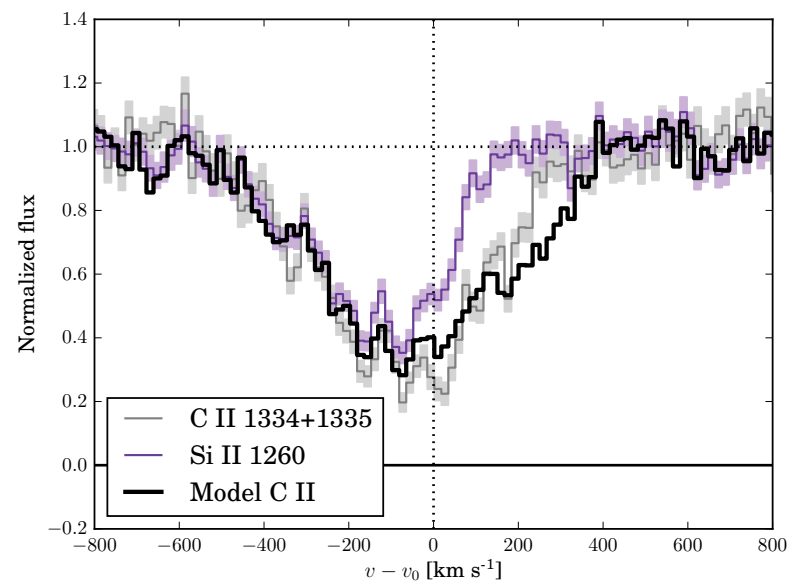

Figure 9. C II 1334, Si II 1260, along with a C II line synthesized from Si II (see text for details).

system of origin, especially those in photo dissociation regions.

Fig. 9 shows a quick back-of-the-envelope check of this hypothesis. Assuming the ions reside in the same physical regions, we used the Si II and a second contribution created by shifting the original profile to the red by the appropriate velocity $\left(\sim 260 \mathrm{~km} \mathrm{~s}^{-1}\right)$ and multiplying it by a free parameter, which was chosen by eye to give a reasonable replication of the observed C II; the chosen value shown in the figure is 0.7 times the original line. It is clear from the figure that the reproduction of the observed C II line is very reasonable, considering that it is generated from a different species with a very different ionization potential.

Whether this is a physically reasonable strength is difficult to say with precision, but we note that to reproduce the feature observed, $\lambda 1334$ must be optically thick and $\lambda 1335$ part transparent. This leaves open a degeneracy of relative level populations and ion abundance, such that the choice of this value only weakly constrains these quantities and thus it is compatible with a wide range of scenarios.

It is also worth noting that C II has a significantly higher ionization potential than Si II, such that the former will be more abundant in regions of higher ionization, with C II in effect tracing slightly different regions than Si II. Furthermore, in these regions of higher ionization, the relative level populations of the Carbon ground state can be significantly altered by shifts in e.g. electron density and radiation field, which can alter the line shape further. We make no attempt at mapping these complex conditions here, but note only that there exists a range of non-exotic physical effects which can generate the deeper and wider $\lambda 1334$ profile we observe while still 
being compatible with the ISM being optically thick in Si II $\lambda 1260$.

\subsection{Conclusion}

In this work, we have re-analyzed an archival FUV HST-COS spectrum of Haro 11 to investigate the kinematics and geometry of both the hot, ionized and cold, neutral ISM along the line of sight to a strongly star forming knot suspected of being the source of the observed LyC leakege from the galaxy. We have used the Apparent Optical Depth method to compute column densities and velocity-binned covering fractions of the gas and compared these results to the extreme cases of either an optically thin, density bounded neutral medium, or a riddled, optically thick, ionization bounded neutral medium. Assuming that the LyC emission previously observed from this galaxy indeed does originate from knot $\mathrm{C}$, we find that the observations are not compatible with the latter case, since the characteristic, bright Lyman- $\alpha$ emission spike at line center is absent in this spectrum. Furthermore, the observations are not consistent with an optically thin, density bounded neutral medium.

We confirm previous authors concluding that the medium is highly ionized with clumps of neutral gas of low velocity-binned covering fraction, increasing the probability of finding direct sight lines to the background star cluster between these. The clumps have HI column densities of gas around $v=0$ in the range $N_{\mathrm{HI}}=6.2_{-1.1}^{+0.9} \times 10^{17} \mathrm{~cm}^{-2}$ given the metallicity of the background H II region, which is likely a slight underestimate of $N_{\mathrm{HI}}$. A conservative estimate of the $\mathrm{H}$ I column density integrated over all velocities is $\log N_{\mathrm{HI}}^{\mathrm{tot}}=19.01 \pm 0.17 \mathrm{~cm}^{-2}$. There is a possibility that the found column densities are in fact lower limits, since the found Si II column densities are close to the limit where this ion becomes optically thick. We therefore conclude that the leaked ionizing photons, if originating from this cluster, most likely escaped via sight lines between the neutral clumps, through an ionized medium which must contain a neutral gas column density $5 \times 10^{13} \lesssim N_{\mathrm{HI}}^{\mathrm{HIS}} \lesssim 4 \times 10^{15} \mathrm{~cm}^{-2}$. This range is bounded downwards by the value at which HI becomes transparent to Lyman- $\alpha$, and upwards by the detectability of Si II in this observation.

It is interesting to note that Haro 11 is relatively on neutral gas poor, and has a very strong ongoing starburst episode. If we were to see a case in which neutral gas depletion and/or ionization provided a densitybounded scenario, this would be a likely place to find it. Still, it seems that dynamical effects of feedback and galaxy-scale interaction plays the greater part in peeling away the neutral gas from the central starbursts and opening passages for $\mathrm{Ly} \alpha$ and $\mathrm{LyC}$ to escape. Given that star formation and merger activity is significantly stronger at $z \gtrsim 1$ than in the local Universe, and that Dark Matter potential wells were shallower, these mechanisms could be even more important in that era. one could speculate that this could provide a piece of the puzzle of which sources have driven the epoch of reionization.

\section{ACKNOWLEDGEMENTS}

The authors thank the anonymous referee for constructive and insightful comments, which have helped improve the quality of this paper significantly.

GÖ \& MH acknowledge the support of the Swedish Research Council, Vetenskapsrådet, and the Swedish National Space Board (SNSB). MH is an Academy Fellow of the Knut and Alice Wallenberg Foundation. This project has made extensive use of the Python-based packages Numpy (Walt et al. 2011), SciPy (Jones et al. 2001-), Matplotlib (Hunter 2007), Pandas (McKinney 2010), LMfit (Newville et al. 2014), and Astropy (Astropy Collaboration et al. 2013).

\section{REFERENCES}

Adamo, A., Östlin, G., Zackrisson, E., et al. 2010, MNRAS, 407,870

Alexandroff, R. M., Heckman, T. M., Borthakur, S., Overzier, R., \& Leitherer, C. 2015, ApJ, 810, 104

Astropy Collaboration, Robitaille, T. P., Tollerud, E. J., et al. 2013, A\&A, 558, A33

Bergvall, N., Zackrisson, E., Andersson, B.-G., et al. 2006, A\&A, 448, 513

Borthakur, S., Heckman, T. M., Leitherer, C., \& Overzier, R. A. 2014, Science, 346, 216
Bouwens, R. J., Illingworth, G. D., Oesch, P. A., et al. 2011, ApJ, 737, 90

Conselice, C. J., Gallagher, J. S., Calzetti, D., Homeier, N., \& Kinney, A. 2000, AJ, 119, 79

Cowie, L. L., Barger, A. J., \& Trouille, L. 2009, ApJ, 692, 1476

Dijkstra, M. 2014, PASA, 31, 40

Erb, D. K. 2016, Nature, 529, 159

Garnett, D. R., Dufour, R. J., Peimbert, M., et al. 1995, ApJL, 449, L77 
Goudfrooij, P., Burgh, E., Aloisi, A., Hartig, G., \& Penton, S. 2010, SMOV: COS NUV Imaging Performance, Tech. rep.

Grimes, J. P., Heckman, T., Strickland, D., et al. 2007, ApJ, 668, 891

Guseva, N. G., Izotov, Y. I., Fricke, K. J., \& Henkel, C. 2012, A\&A, 541, A115

Hayes, M. 2015, PASA, 32, e027

Hayes, M., Östlin, G., Atek, H., et al. 2007, MNRAS, 382, 1465

Hayes, M., Östlin, G., Mas-Hesse, J. M., \& Kunth, D. 2009, AJ, 138, 911

Heckman, T. M., Alexandroff, R. M., Borthakur, S., Overzier, R., \& Leitherer, C. 2015, ApJ, 809, 147

Heckman, T. M., Borthakur, S., Overzier, R., et al. 2011, ApJ, 730, 5

Henry, A., Scarlata, C., Martin, C. L., \& Erb, D. 2015, ApJ, 809, 19

Holland, S. T., et al. 2014, Cosmic Origins Spectrograph Instrument Handbook, 6th edn., Baltimore: STScI

Hunter, J. 2007, Computing in Science Engineering, 9, 90

Iwata, I., Inoue, A. K., Matsuda, Y., et al. 2009, ApJ, 692, 1287

Izotov, Y. I., Orlitová, I., Schaerer, D., et al. 2016a, Nature, 529,178

Izotov, Y. I., Schaerer, D., Thuan, T. X., et al. 2016b, MNRAS, 461, 3683

James, B. L., Aloisi, A., Heckman, T., Sohn, S. T., \& Wolfe, M. A. 2014, ApJ, 795, 109

Jaskot, A. E., \& Oey, M. S. 2013, ApJ, 766, 91

Jones, E., Oliphant, T., Peterson, P., et al. 2001-, SciPy: Open source scientific tools for Python, , , [Online; accessed 2014-11-25]

Jones, T. A., Ellis, R. S., Schenker, M. A., \& Stark, D. P. 2013, ApJ, 779, 52

Kunth, D., Mas-Hesse, J. M., Terlevich, E., et al. 1998, Astronomy and Astrophysics, 20, 11

Leitet, E., Bergvall, N., Hayes, M., Linné, S., \& Zackrisson, E. 2013, A\&A, 553, A106

Leitet, E., Bergvall, N., Piskunov, N., \& Andersson, B.-G. 2011, A\&A, 532, A107

Leitherer, C., Hernandez, S., Lee, J. C., \& Oey, M. S. 2016, ApJ, 823, 64
McKinney, W. 2010, in Proceedings of the 9th Python in Science Conference, ed. S. van der Walt \& J. Millman, 51 $-56$

Nestor, D. B., Shapley, A. E., Kornei, K. A., Steidel, C. C., \& Siana, B. 2013, ApJ, 765, 47

Newville, M., Stensitzki, T., Allen, D. B., \& Ingargiola, A. 2014, LMFIT: Non-Linear Least-Square Minimization and Curve-Fitting for Python $\uparrow$, , , doi:10.5281/zenodo.11813

Östlin, G., Amram, P., Bergvall, N., et al. 2001, A\&A, 374, 800

Östlin, G., Hayes, M., Kunth, D., et al. 2009, AJ, 138, 923

Östlin, G., Marquart, T., Cumming, R. J., et al. 2015, A\&A, 583, A55

Overzier, R. A., Heckman, T. M., Tremonti, C., et al. 2009, ApJ, 706, 203

Pardy, S. A., Cannon, J. M., Östlin, G., Hayes, M., \& Bergvall, N. 2016, ArXiv e-prints, arXiv:1609.04020

Pettini, M., Rix, S. A., Steidel, C. C., et al. 2002, ApJ, 569, 742

Prochaska, J. X., Kasen, D., \& Rubin, K. 2011, ApJ, 734, 24

Quider, A. M., Pettini, M., Shapley, A. E., \& Steidel, C. C. 2009, MNRAS, 398, 1263

Rivera-Thorsen, T. E., Hayes, M., Östlin, G., et al. 2015, ApJ, 805, 14

Robertson, B. E., Furlanetto, S. R., Schneider, E., et al. 2013, ApJ, 768, 71

Sandberg, A., Östlin, G., Hayes, M., et al. 2013, A\&A, 552, A95

Savage, B. D., \& Sembach, K. R. 1991, ApJ, 379, 245

Scarlata, C., \& Panagia, N. 2015, ApJ, 801, 43

Siana, B., Teplitz, H. I., Ferguson, H. C., et al. 2010, ApJ, 723,241

Vader, J. P., Frogel, J. A., Terndrup, D. M., \& Heisler, C. A. 1993, AJ, 106, 1743

Vanzella, E., Giavalisco, M., Inoue, A. K., et al. 2010, ApJ, 725,1011

Vanzella, E., Guo, Y., Giavalisco, M., et al. 2012, ApJ, 751, 70

Verhamme, A., Orlitová, I., Schaerer, D., \& Hayes, M. 2015, A\&A, 578, A7

Walt, S. v. d., Colbert, S. C., \& Varoquaux, G. 2011, Computing in Science \& Engineering, 13, 22

Zackrisson, E., Inoue, A. K., \& Jensen, H. 2013, ApJ, 777, 39 